Thorax (1954), 9, 340.

\title{
EXTRA-AURICULAR MITRAL VALVOTOMY
}

\author{
BY \\ HELGE BADEN AND FREDERIK THERKELSEN \\ From Surgical Department $D$ and Surgical Department $R$, Rigshospitalet, Copenhagen, Denmark
}

(RECEIVED FOR PUBLICATION JUNE 8, 1954)

To-day the routine approach to the mitral orifice is through the left auricular appendage, approximately the same route that Souttar (1925) used in the first successful valvotomy. The transventricular route has been abandoned. It is evident, e.g. from Smithy, Boone, and Stallworth's (1950) reports, that this method, besides being dangerous, affords unsatisfactory results. At times, however, the anatomical conditions make it impossible to use the auricular approach. In such cases another part of the atrium may be entered, and it is not necessary to refrain from operating as was done in two of the cases of Andrus, Blalock, and Milnor (1953) and in one of those of Wulff, Biörck, Bergh, Krook, Axén, and Lundskog (1953).

Until Bailey, Olsen, Keown, Nichols, and Jamison in 1952 adopted intermittent occlusion of the carotid arteries in the prophylaxis against cerebral embolism during valvotomy, they regarded appendageal thrombi as a contraindication to using the auricular approach to the atrium. For this reason, they used an extra-auricular approach in 24 cases. In three they entered through the atrial wall proper. Although all three operations were successful, they considered this entrance too dangerous and have abandoned it. In 21 cases they entered through the left superior pulmonary vein. No embolism occurred in these cases, but two deaths were ascribed to the technique. According to Bailey and others, the last-mentioned procedure involves risks: a reduced flow of blood to the heart during the operation, atypical solitary entrance of the pulmonary vein, difficulty in reaching down to the mitral orifice, which, combined with the narrow access, may easily lead to rupture of the vein, and finally the risk of thrombosis at the site where the vein has been sutured.

In our opinion, thrombi in the auricle do not necessitate the extra-auricular approach, as we are not convinced that it guards against embolism. Recently we introduced pre- and post-operative administration of dicoumarol in order to prevent the formation of fresh thrombi. This is expected to acquire particular importance in cases with a history of embolism or of auricular fibrillation. The treatment was introduced by Dr. O. Storm, who is also in charge of the daily control of the patients. On the day of the operation the patients have prothrombin values close to $20 \%$, and the few cases so far treated have not exhibited any increased tendency to bleed during or after the operation. Dicoumarol medication must be under painstaking control, and accurate laboratory analyses are of decisive importance. (A special report on dicoumarol treatment in these cases is being prepared.)

In the three cases to be reported below, the auricular approach could not be used. Two patients had no auricular appendage. In one of these cases valvotomy had been given up in another hospital, as the base of the appendage would not admit a finger. The operation was therefore restricted to amputation of the auricle. The second patient had two years previously had a mitral valvotomy and amputation of the auricular appendage in our hospital. In the third case the valvotomy was done at the same time as an operation on the right lung. As in a similar case reported by Shaw (1953), the finger was introduced into the atrium through the right inferior pulmonary vein.

In all three cases a satisfactory "finger fracture" valvotomy was performed, and there were no operative deaths.

\section{CASE RePORTS}

CASE 1.-A man, aged 40, from 1933 had become increasingly incapacitated by mitral stenosis. In 1951 mitral valvotomy was attempted in another hospital, but it was given up, as the auricle was too narrow to admit a finger.

On admission here in 1953 his main complaints were pronounced dyspnoea on exertion, cough and expectoration, irregular action of the heart, and violent paroxysms of palpitations. The patient had never had crucial pulmonary oedema, haemoptysis, or signs of right heart failure. Digitalis had been given constantly. The nutritional condition was fair, and at rest there was no dyspnoea or cyanosis of the lips. At the apex, the first heart sound was accentuated and there was an 
occasional presystolic roulement. A faint, rumbling diastolic murmur was heard, but there was no systolic murmur. The pulmonary second sound was split, and was louder than the aortic second sound. There was no sign of congestion. At rest, the pressure in the pulmonary artery was $60 \mathrm{~mm}$. Hg systolic and 42 diastolic; the mean pressure in the pulmonary capillaries was $32 \mathrm{~mm}$. at rest, the cardiac output at rest $5.281 . / \mathrm{min}$. The P.C.V. curve had a single peak and rather pronounced excursions. Teleradiography showed a slightly enlarged heart of mitral configuration.

On March 20, 1953, mitral valvotomy was performed. The pericardium was opened anterior to the phrenic nerve. There was almost total adhesion between the pericardial layers, and the heart had to be exposed by sharp dissection. The auricular appendage was absent, but a few knots of suture were palpable at its site. A purse-string suture was applied to the area between the site of the auricle and the left superior pulmonary vein, so that the horizontal branch of the coronary artery was free. Outside the purse-string suture two rows of stay sutures were applied, four in each row, one row on each side of the site to be incised (Fig. 1). Through a suitable incision in this site, the ungloved index finger was

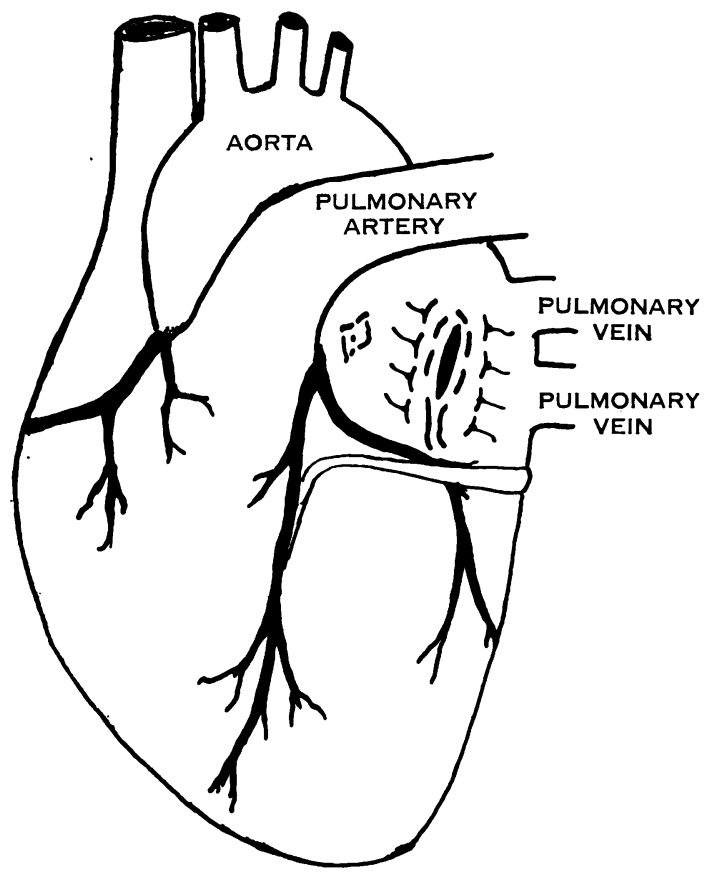

FIG. 1.-Schematic representation of the extra-auricular approach to the mitral orifice.

introduced through a thick layer of trabeculae. There was no regurgitation. The cusps of the valves, particularly the medial one, were rather markedly calcified, and the orifice admitted only the tip of the finger. After both commissures had been completely opened, the orifice admitted about two fingers. No regurgitation occurred. An attempt was made to tighten the pursestring suture, but it broke. As the finger was withdrawn, the stay sutures were tied in pairs. There was almost complete haemostasis; only inferiorly, the incision had been torn a little, and to be on the safe side we applied a few atraumatic silk sutures at this and a few other sites. Haemostasis was complete, the pressure in the atrium felt lower than before; the pericardium was partially closed, and the chest wound closed with underwater drainage.

The post-operative course was uneventful apart from a small pulmonary infarct. Six months after the operation the patient had not had pulmonary oedema or cough; he could walk an unlimited distance at a normal pace and had been working in his garden. He was still taking digitalis. He looked well. There was a grade 1 systolic and a grade 1 diastolic rolling murmur at the apex. The first heart sound was accentuated. The pulmonary second sound was accentuated and split. The auricular fibrillation and teleradiographic appearances were unchanged.

CASE 2.-A man, aged 41 , in 1950 had had a mitral valvotomy in the department. At that time the mitral orifice just admitted the tip of the index finger. The edges were thickened, fibrous, and slightly calcified; there was some regurgitation. When an attempt was made to split the commissures, the valves yielded like elastic, and great force was required in order gradually to divide the antero-lateral commissure in its entire length. Then the orifice admitted a whole finger without difficulty; the regurgitation remained undiminished.

During the first 18 months after this operation the patient reported marked subjective improvement, but after an attack of pneumonia he began to go downhill. $\mathrm{He}$ had increasing dyspnoea on exertion, palpitations, and nocturnal attacks of dyspnoea. After having been admitted several times to other hospitals, he returned to us in the autumn of 1953 .

Auscultation of the heart revealed, at the apex, a grade 3 systolic and a grade 2 proto-mesodiastolic murmur. The first heart sound was not accentuated, but the second sound was split. The pulmonary second sound was louder than the aortic second sound. The heart action was slow and irregular. As previously, the radiographs showed a heart of mitral configuration, but not enlarged. Catheterization of the heart disclosed a moderately increased pressure, increasing on exertion. The P.C.V. curve did not suggest insufficiency.

It was concluded that the previous valvotomy had not been sufficiently radical or that a recurrence had been caused by the pneumonia, so it was decided to operate again.

On August 11, 1953, mitral valvotomy was performed. The pericardium was a few millimetres thick, and the pericardial sac was obliterated. Where the pericardium had been left open there was a delicate fibrous membrane. The entire heart was exposed so that massage might be applied, if necessary. The superior pulmonary vein was isolated. It was as thick as a thumb and rather short. The distance down to the valves seemed long. There 
was a small prominence on the atrium, where the auricular appendage had been. On each side a row of $U$ sutures was applied to the atrial wall. The ungloved index finger, armed with Holmes Sellors's (1951) " fingernail knife," was introduced through an incision of suitable size. Crossing of the $U$ sutures afforded excellent haemostasis. The mitral orifice was oval, admitting only the tip of the finger, at an estimate $3 \times 5 \mathrm{~mm}$. wide. The edges were rather calcified, and there was only slight regurgitation; the valves were not drawn downwards. The opening was to the anterior, lateral aspect, so there was not much of the antero-lateral commissure to be split. The postero-medial commissure, on the other hand, could be split all the way down to the mitral ring, thanks to the " finger-nail knife." Thereafter, the orifice admitted one and a half index fingers. The sub-valvular apparatus was only moderately changed. As the finger was withdrawn, the stay sutures were crossed, stopping the haemorrhage entirely. Interrupted sutures were applied, and then the stay sutures were tied. The pericardium was left open.

The post-operative course was uneventful, and the patient was feeling relatively well on discharge. He has not yet returned for a follow-up examination.

CASE 3.-A 41-year-old woman, who was receiving a disability pension, had a history of rheumatic fever in 1924. From 1949 she had been suffering from increasing dyspnoea on exertion, palpitations, oedema of the ankles, and paroxysms of dyspnoea. In 1950 she suffered infarction of the kidney and spleen and mild cerebral embolism. Periodically she had courses of digitalis. After a pulmonary infarct in 1953 she developed an abscess in the lower lobe of the right lung. After about six weeks' conservative treatment elsewhere she was transferred to us.

On admission she was ill and anaemic, showing slight dyspnoea and cyanosis of the lips at rest. At the apex a grade 2 blowing systolic murmur followed a snapping first sound and a holodiastolic rumbling murmur. Over the aorta a systolic murmur of grade 2-3 was heard. No capillary pulsation was visible. The radial pulse was slow and weak. An E.C.G. showed fibrillo-flutter and right-sided axis deviation. Teleradiography revealed a moderately enlarged, typical mitral heart and, in the basal part of the right lung field, a dense shadow and fluid level. The diagnosis was mitral stenosis, pulmonary abscess, and a less significant combined aortic stenosis and insufficiency. Treatment for three weeks with postural drainage, bronchoscopic aspirations, and antibiotics was of little effect.

On February 4, 1954, right-sided inferior lobectomy and mitral valvotomy were performed. The chest was opened on the right side. In the lower lobe and between the latter and the diaphragm there was an abscess, the size of an orange, that burst while it was being detached. The branches of the pulmonary artery to the apical segment of the lower lobe and to the remaining parts of the lobe were ligated and stitched separately. The bronchus was divided just below the origin of the middlelobe bronchus, closed with atraumatic silk by the method of Sweet and later covered with a flap of the pericardium.
The right inferior pulmonary vein was exposed as far peripherally as possible and divided between Péan's forceps. The pericardium was opened around the vein, which could then be completely isolated. To be on the safe side, we applied five pairs of stay sutures, one row on each side of the vein, and in addition a pursestring suture at its base. After applying a Satinsky forceps, the ungloved right index finger could be introduced into the very large left atrium. At the outset, only the finger-tip was admitted, but after its introduction the opening was cut somewhat wider so that it could admit a sufficient portion of the finger. The outlet of the mitral valves was shrunk and there was pronounced calcification along the edges of the cusps. The orifice admitted only the tip of the index finger. The medial as well as the lateral commissure was easy to split all the way to the fibrous ring. The sub-valvular apparatus was shrunk, thickened, and somewhat brittle. Now the orifice admitted one and a half fingers. There was some regurgitation before the valvotomy, less after. The finger was withdrawn while Satinsky's forceps was applied and the purse-string suture tied. This gave complete haemostasis. Even so, an extra, continuous atraumatic silk suture was applied over the edges. The stay sutures were not used and removed again. The patient was awake immediately after the operation was over. The drainage tube was removed on the fourth day, and the post-operative course has been uneventful.

\section{Discussion}

With the increasing number of operations for mitral stenosis, the surgeon cannot avoid coming across cases in which the routine approach to the left atrium through the auricular appendage is inapplicable. This occurred in three of our 115 cases. As mentioned above, Bailey and others (1952) have three times successfully entered the atrial wall proper in order to avoid liberating emboli from a thrombosed auricle.

Bailey and others, however, fear that the pursestring suture will tear and cause uncontrollable haemorrhage. They have therefore abandoned the method. We feel that a purse-string suture around an incision which is about $3 \mathrm{~cm}$. in length will easily tear or burst. In our Case 1 we applied two rows of stay sutures, five on each side of the incision, and central to these a purse-string suture (Fig. 1). After introducing the finger we kept the atrial wall leak-tight by tightening the purse-string suture. When the finger was withdrawn, we tried to close the wound by tying the purse-string suture, but it burst. The wound was then closed by tying the stay sutures in pairs. In Case 2, only one row of stay sutures was applied on each side of, and parallel to, the site of incision. After the atrial wall was opened and the finger introduced, the stay sutures were crossed, so that the atrial wall fitted the finger snugly. After the finger had been withdrawn 
the stay sutures were kept crossed, and the incision was closed with a row of interrupted sutures, the stay sutures being tied last. This method afforded satisfactory haemostasis and closure, but it required vigilance while the finger was in the atrium, and the crossed stay sutures impeded the manipulations.

In both instances an easy and undramatic closure was effected with the stay sutures. It is an advantage, however, to have a purse-string suture as well (Case 1), as the manipulations within the heart involved less bleeding when the pursestring suture was tightened a little.

In the third case, approached from the right, after inferior lobectomy, we had applied two rows of stay sutures and a purse-string suture around the base of the inferior pulmonary vein. As the purse-string suture afforded excellent closure, the stay sutures could be removed again.

\section{SUMMARY}

Three mitral valvotomies, performed without introducing the finger through the auricular appendage, are described. The appendage had been removed at an earlier operation in two cases. In one case a right lower lobectomy and mitral valvotomy were carried out in one sitting by entering the atrium through the right inferior pulmonary vein. It is felt that the easy and undramatic course of the operations through the atrial wall largely depends on the development of a special technique for the closure. A purse-string suture is used to secure a tight fit around the finger during the finger fracture while the actual closure is affected with two rows of stay sutures, placed parallel to the incision and tied in pairs as the finger is withdrawn.

\section{REFERENCES}

Andrus, E. Cowles, Blalock, A., and Milnor, W. R. (1953). Arch. Surg., 67, 790.

Bailey, C. P., Olsen, A. K., Keown, K. K., Nichols, H. T., and Jamison, W. L. (1952). J. Amer. med. Ass., 149, 1085.

Sellors, T. Holmes (1951). Lancet, 1, 448.

Shaw, K. M. (1953). Ibid., 2, 1130.

Smithy, H. G., Boone, J. A., and Stallworth, J. M. (1950). Surg. Gynec. Obstet., 90, 175.

Souttar, H. S. (1925). Brit. med. J., 2, 603.

Wulff, H. B., Biörck, G., Bergh, N. P., Krook, H., Axén, Ø., and Lundskog, O. (1953). Acta med. scand., 144, 275.

\section{AdDendum}

Since this report was submitted for publication, we have done four more extra-auricular mitral valvotomies. In three cases we went through the atrial wall proper using the technique described above. The indications were very small auricular appendages and/or appendages completely obliterated by organized thrombotic material. The operations and the post-operative course were uneventful in all three cases. On the fourth patient a right lower lobectomy for carcinoma of the lung was performed, and the mitral valve was approached through the right lower pulmonary vein. The operation was uneventful, but the patient died suddenly two hours after the operation. The necropsy offered no explanation for the cause of death. 